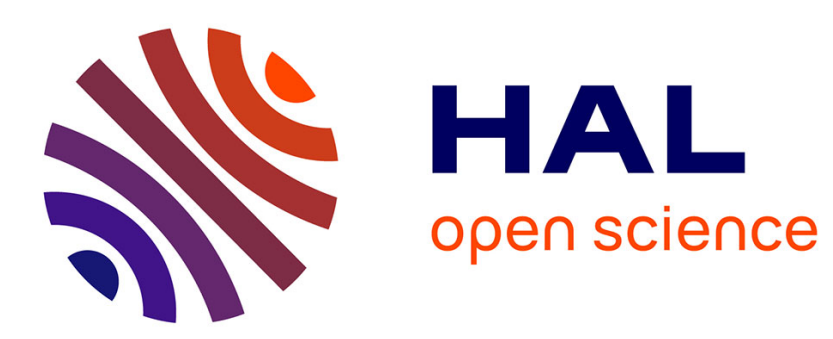

\title{
Automated registration of 3D x-ray angiography images to magnetic resonance images
}

\author{
Erwan Kerrien, Olivier Levrier, Rene Anxionnat, Sylvain Sauty, Jerome
}

Knoplioch

\section{- To cite this version:}

Erwan Kerrien, Olivier Levrier, Rene Anxionnat, Sylvain Sauty, Jerome Knoplioch. Automated registration of 3D x-ray angiography images to magnetic resonance images. SPIE Medical Imaging 2001, SPIE - The International Society for Optical Engineering, Feb 2001, San Diego, United States. pp.623632, 10.1117/12.431136 . hal-01819796

\section{HAL Id: hal-01819796 \\ https://hal.science/hal-01819796}

Submitted on 21 Jun 2018

HAL is a multi-disciplinary open access archive for the deposit and dissemination of scientific research documents, whether they are published or not. The documents may come from teaching and research institutions in France or abroad, or from public or private research centers.
L'archive ouverte pluridisciplinaire $\mathbf{H A L}$, est destinée au dépôt et à la diffusion de documents scientifiques de niveau recherche, publiés ou non, émanant des établissements d'enseignement et de recherche français ou étrangers, des laboratoires publics ou privés. 


\title{
Automated registration of 3D X-ray angiography images to magnetic resonance images
}

\author{
E. Kerrien ${ }^{a}$, O. Lévrier ${ }^{b}$, MD, R. Anxionnat ${ }^{c}$, MD, S. Sauty ${ }^{a}$ \\ and J. Knoplioch ${ }^{a}$ \\ ${ }^{a}$ GE Medical Systems, Buc, France \\ ${ }^{b}$ Hôpital La Timone, Marseille, France \\ ${ }^{c}$ Hôpital neurologique, Nancy, France
}

\begin{abstract}
An automated algorithm for frameless registration of intra-cranial 3D X-ray angiograms (3DXA) to Magnetic Resonance (MR) images is described and evaluated. The registration procedure starts with the manual designation of a pre-defined anatomical point in both modalities. Then, the registration is performed through an iterative process that alternatively estimates the rotation and translation using an original correlation optimization scheme. The evaluation procedure implied comparisons with both manual (11 cases) and stereotactic frame based ( 9 cases) registration. The results encompass that manual registration is not a viable method of reference for registering such volumes whereas stereotactic frame (or equivalent means) is acceptable for validation purpose. A maximum error of $4 \mathrm{~mm}$ was measured for our automated algorithm while variations of up to $5 \mathrm{~mm}$ were considered on the initial point location. Convergence time was below 1 minute while an average time of 30 minutes was required to perform manual registration. This validation procedure demonstrates a good precision for the automated algorithm when compared to a stereotactic frame based matching. Such an algorithm could make the intra-cranial pathology assessment more reliable, enable frameless radiotherapy planning of AVMs in 3D, ease biopsy planning in neurosurgery or be helpful for educational purpose.
\end{abstract}

Keywords 3D X-ray angiography, Magnetic Resonance Imaging, registration

\section{Introduction}

In the course of their dayly practice, interventional neuroradiologists and neurosurgeons make use of many different imaging modalities to diagnose pathologies, treat them and follow the patients up. In particular, the vascular pathologies, such as aneurysms, arterio-venous malformations (AVM) or fistulae, or related pathologies, such as intra-cranial tumors, demand that the surgeon has a precise knowledge of the vasculature, the tissues surrounding the pathology and the functional role of these regions. Since no single modality is able to provide all this information at once, the simultaneous analysis of multiple types of images is required. As a result, the need for registration algorithms is becoming more and more crucial for the healthcare community, especially for the management of neuropathologies.

Digital Subtracted Angiography (DSA) images are the cornerstone of interventional neuroradiology. Lately, neuroradiologists have been using in clinical routine Three-dimensional X-ray Angiography (3DXA) images of the brain vasculature. Such volumetric images have been proven to bring an actual supplementary help to the physician [1,2]. Even though some studies are still needed, many previous works are available in a view to perform the registration of a DSA image with a 3DXA volume $[3,4,5,6,7]$. While DSA and 3DXA provide very precise and complete information on both the anatomo-morphology of the vasculature and the hemodynamics, no knowledge of the intra-cranial tissues can be extracted from such images.

On the other hand, Magnetic Resonance (MR) imaging is a powerful and unique tool that provides images with a very good spatial resolution and an excellent contrast on the soft tissues of the brain. However, the analysis of the vasculature is

Proceedings of SPIE Medical Imaging 2001, K. Janson Ed., 18-22 February 2001, San Diego, vol. 4322 part 1, pp. 623-632. 
hindered by distortions, flow effects and the spatial resolution is still not high enough. Dedicated imaging protocols were developed to tackle part of these problems (Magnetic Resonance Angiography, MRA) but the price for these improvements is the loss of contrast on the brain tissue.

The ability to register a 3DXA volume and an MR exam would therefore help perform an accurate diagnosis of a vascular disease and provide complete information to the neurosurgeons to treat it. Furthermore, numerous works describe ways to match MRI data with Computed Tomography (CT) volumes, Positron Emission Tomography (PET) or Single Photon Emission Computed Tomography (SPECT) acquisitions and intra-cranial Echography (US) images. As a consequence, the MR modality appears as a footbridge to be used to match many imaging modalities, including 3DXA.

\section{Material and Methods}

\subsection{Images}

3DXA volumes are reconstructed using a tomography algorithm based on a set of about 50 DSA images. This set of images is acquired on a standard open C-arm (LCN+, GE Medical Systems) performing a rotation of about 200 degrees around the patient's head while a contrast dye is injected in the artery of interest. Reconstructions of both internal carotid and vertebral arteries are being considered.

The choice for the MR acquisition protocol was guided by two constraints:

- in order for such images to be of interest, they have to show a good contrast for the brain soft tissues ;

- since a 3DXA volume only depicts the vasculature depending on the injected artery, the best possible accuracy on the brain vasculature was looked for so as to provide the registration algorithm with some information common to both modalities under study.

As previously stated, MRA cannot comply with the first requirement. As a result, we elected a T1 weighted protocol (giving good contrast on the brain tissue) enhanced by an intra-venous injection of gadolinium (highlighting the vasculature). MR images were acquired on two different systems (Signa 1.5T, GE Medical Systems and Magnetom Symphony, Siemens AG) to account for possible variations in image characteristics. High resolution images (512x512 or 256x256 matrix with a slice thickness of $1.5 \mathrm{~mm}$ ) were used.

\subsection{Registration techniques}

Distortions in MR are known to be a potential problem. However, since the imaged portion of the body is relatively small (the head), we chose to neglect their possible impact on the registration. This mono-patient registration problem can therefore be seen as the search for a rigid-body transform. Basically, three groups of methods can be looked over: point-based, featurebased and voxel-based registration algorithms.

The use of external markers presents many drawbacks, especially in terms of a higher complexity for the data acquisition and the impossibility to perform retrospective registration. On the other side, manual registration is particularly tedious in our case due to the difficulty to identify vessels in MR.

Since only blood vessels are visible in a 3DXA volume, a feature-based registration method would require the segmentation of vessels in both modalities. If this step would be rather straightforward in 3DXA, the difficulty of the task in a T1 weighted MR image is demonstrated by the lack of scientific work concerning this MR protocol on one side, and, on the other side, the many different solutions studied along the past few years to achieve this goal in MRA acquisitions, which yet depict the blood vessels in a rather clear manner $[8,9,10,11]$.

Different robust similarity measures have been defined whose power has been demonstrated for registering MR, CT, PET and SPECT images [12, 13, 14, 15]. If these modalities show sometimes important discrepancy, the depiction of global structures such as the skull, the cortex or the ventricles in all of them backs up the efficacy of such criteria in the neuro case. Similarity measures seemed the best available methods to perform 3DXA/MR registration. However, 3 problems had to be solved:

Proceedings of SPIE Medical Imaging 2001, K. Janson Ed., 18-22 February 2001, San Diego, vol. 4322 part 1, pp. 623-632. 
- initialization: the vasculature is literally drowned amidst the mass of information regarding soft tissues in MR, which requires a hint about where to find it in this modality;

- convergence: the tree-shape of the vasculature generates numerous local optima for the similarity measure;

- time: 3DXA volumes are coded on a $512 \times 512 \times 512$ voxels cube and MR volumes are composed of either 124 or 174 images which are coded on a $512 \times 512$ or $256 \times 256$ matrix. This huge volume of data had to be processed in a very short time (less than $3 \mathrm{mn}$ ) for the algorithm to be acceptable in a clinical environment.

In the next section, the algorithm we designed to overcome these problems is described. Section 4 reports the study that was made to validate the use of a stereotactic frame and to reject the manual registration as a method of reference for the matching of a 3DXA volume with an MR acquisition. Section 5 provides the results of the evaluation of our registration algorithm in comparison with the technique using a stereotactic frame.

\section{Registration algorithm}

\subsection{Initialization and hypothesis}

The identification of a vessel bifurcation on the circle of Willis is very easy in MR as well as in 3DXA. This provides a fast and easy way to initiate the registration: the internal carotid bifurcation must be indicated in the case where the internal carotid artery is shown on the 3DXA volume, and the tip of the basilar tree has been chosen in the case where a vertebral artery was injected.

As a result, we can consider we have a good estimation of the translation part of the registration: an error of less than 3 $\mathrm{mm}$ should affect it (the size of the internal carotid artery is about 4-5 mm). Besides, the rotation is unknown but, except for very exceptional cases, its angle should be within \pm 30 degrees.

Two other problems need to be addressed. On one hand, the convergence properties suffer from the sparseness of the vascular information in the volumes. On the other hand, the convergence time is likely to be long, due to the amount of data to process. As a consequence, the philosophy that we followed in designing the algorithm consisted in reducing the amount of data, through reducing their dimensionality, while preserving the relevant information.

\subsection{Rotation estimation}

Let's first assume that the translation is perfectly known through the knowledge of a pair of homologous points in both modalities (user-defined). The rotation remains to be recovered. Let's call a thick shell any set of voxels located between two spheres centered on the homologous pair of points. Any such set is globally invariant under any rotation centered on this pair of points. We extract in both modalities one such set which we truncate by removing a cone in the direction of the nose: sinuses show a contrast uptake in MR, which creates a large zone in hypersignal that attracts the arteries in the optimization process. Figure 1 depicts such thick shells in a 3DXA and an MR volumes.

In both sets of voxels, arteries appear in hypersignal. In a second step, a radial Maximum Intensity Pixel (MIP) projection of each thick shell onto their external surface is performed (figure 2(a)). These two steps allow for a dramatic reduction of data (voxels selection in a thick shell and projection onto a surface) while preserving relevant information (radial MIP projection).

A planar mapping of the external spheres was used to store the information resulting from the MIP projection as regular images. Each truncated sphere can be spanned by two angles: $\theta$ varying in $[-\pi, \pi]$ around the $\mathrm{x}$ axis and $\varphi$ varying in $\left[0, \varphi_{\max }\right]$ (see figure 2(b)). A straightforward representation would be to define pixel coordinates $(i, j)$ such that $i \propto \theta$ and $j \propto \varphi$. This representation entails important distortions and, as a result, disbalances the amount of contribution of each area of the image in the calculations of the similarity criterion. A so-called "polar" representation was preferred: the origin being at the center of the image, $\theta$ is the polar angle in the image plane and $\varphi$ is proportional to the modulus (see figure 2(b)).

Proceedings of SPIE Medical Imaging 2001, K. Janson Ed., 18-22 February 2001, San Diego, vol. 4322 part 1, pp. 623-632. 


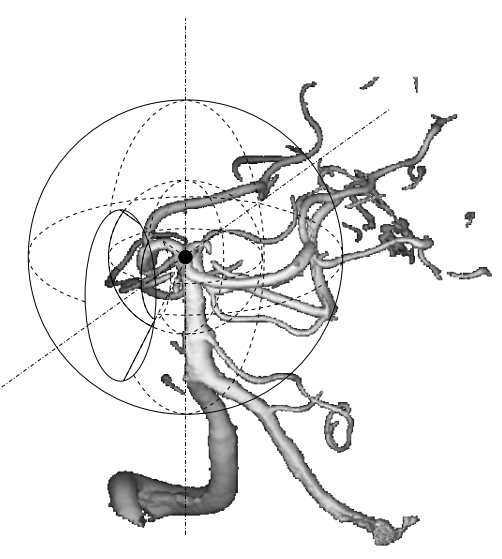

(a) 3DXA

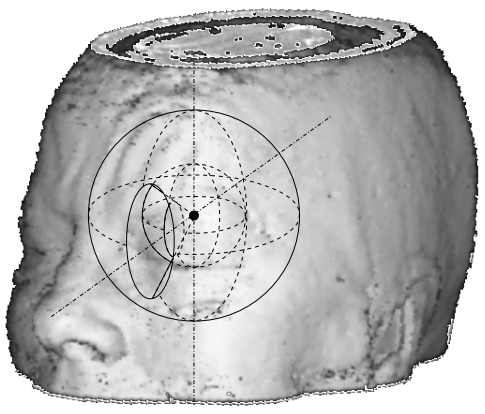

(b) MR

Figure 1: Thick shell extraction

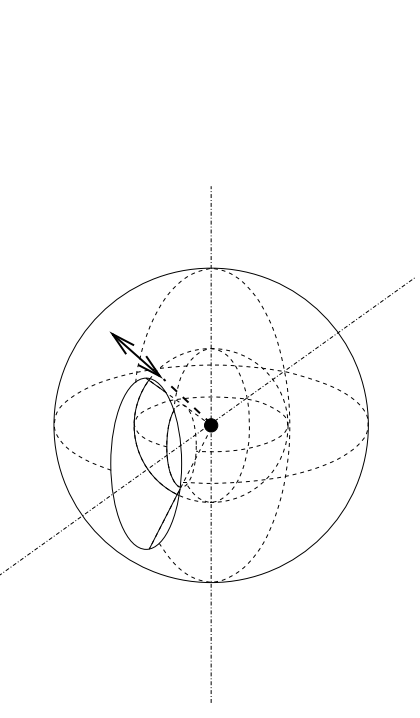

(a) MIP projection of a thick shell on its external surface

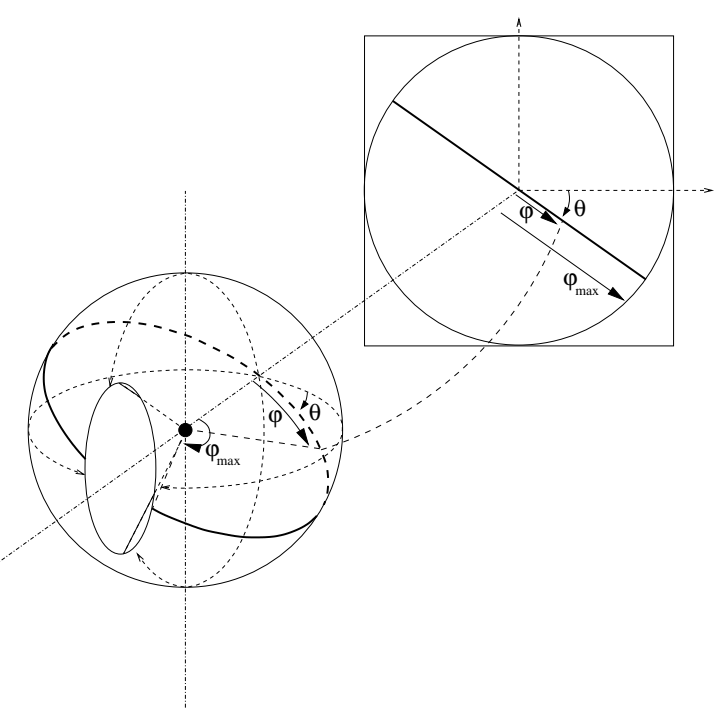

(b) Mapping of the external surface onto a plane

Figure 2: Mapping of a thick shell onto a plane vol. 4322 part 1, pp. 623-632. 


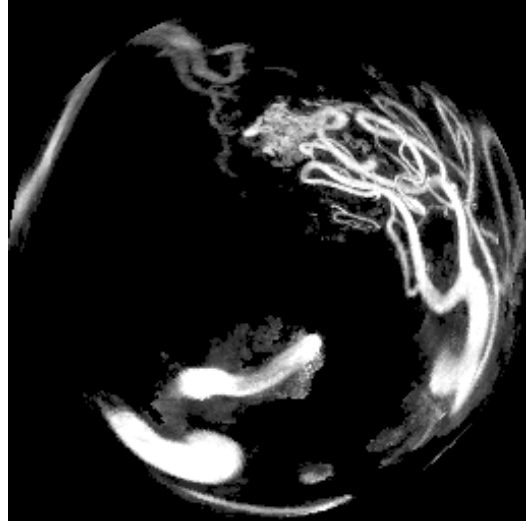

(a) 3DXA

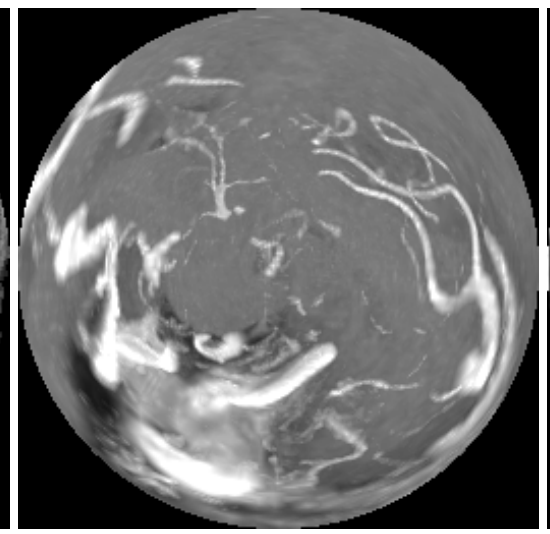

(b) MR

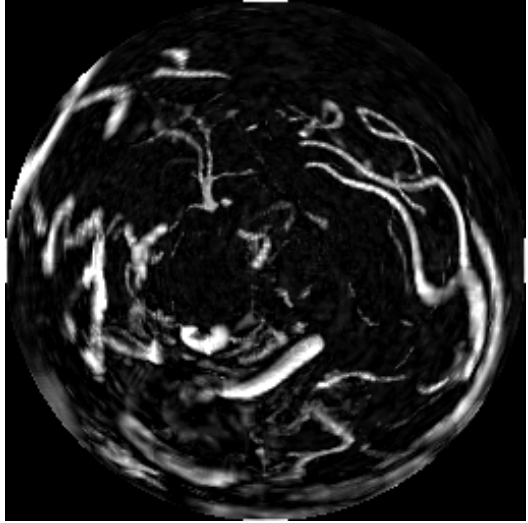

(c) MR after tophat

Figure 3: Example results of the mapping of thick shells in 3DXA and MR

If we consider a point $P=(x, y, z)$ on the external sphere. The radius of the sphere is unimportant. As a result, the corresponding pixel $(i, j)$ is determined by ( $N$ is the size of the image in pixels):

$$
\left\{\begin{array} { r l } 
{ x } & { = - \operatorname { c o s } \varphi } \\
{ y } & { = - \operatorname { s i n } \varphi \operatorname { c o s } \theta } \\
{ z } & { = \operatorname { s i n } \varphi \operatorname { s i n } \theta }
\end{array} \Leftrightarrow \left\{\begin{array} { r l } 
{ \varphi } & { = \operatorname { a r c c o s } ( - x ) } \\
{ \operatorname { c o s } \theta } & { = - \frac { y } { \operatorname { s i n } \varphi } } \\
{ \operatorname { s i n } \theta } & { = \frac { z } { \operatorname { s i n } \varphi } }
\end{array} \Leftrightarrow \left\{\begin{array}{rl}
i & =\frac{N}{2}\left(1+\frac{\varphi}{\varphi_{\max }} \cos \theta\right) \\
j & =\frac{N}{2}\left(1-\frac{\varphi}{\varphi_{\max }} \sin \theta\right)
\end{array}\right.\right.\right.
$$

In the case where $\varphi=0, \cos \theta$ and $\sin \theta$ are undefined but the singularity is easily overcome by setting the corresponding pixel at the center of the image

The inverse formulae can be determined:

$$
\exists P \text { on the external sphere } \Leftrightarrow\left\{\rho^{2}=\left(\frac{2 i}{N}-1\right)^{2}+\left(\frac{2 j}{N}-1\right)^{2}\right\}<1 \Rightarrow\left\{\begin{aligned}
\varphi & =\rho \varphi_{\max } \\
\cos \theta & =\frac{1}{\rho}\left(\frac{2 i}{N}-1\right) \\
\sin \theta & =\frac{1}{\rho}\left(\frac{2 j}{N}-1\right)
\end{aligned}\right.
$$

Once again, these formulae are not determined at the center of the image $(\rho=0)$ but the case is easily overcome.

These direct and inverse formulae allow for a simple calculation of the warping implied in the image plane by a rotation of the external sphere: pixel $(i, j)$ in the image corresponds to point $(x, y, z)$ (inverse formulae) which is rotated to point $\left(x^{\prime}, y^{\prime}, z^{\prime}\right)$ which itself corresponds to pixel $\left(i^{\prime}, j^{\prime}\right)$ (direct formulae).

An example of the images obtained through this process is shown on figure 3(a) and 3(b): they appear as seen by an observer with a $2 \varphi_{\max }$ vision angle, located at the center of the thick shells. We can see that the images are still somewhat different. In order to enhance their similarity, a simple tophat operator is applied, leading to images as shown on figure 3(c). The rotation is recovered by optimizing the centered normalized cross-correlation between the images, while spanning warpings corresponding to rotations in the $3 \mathrm{D}$ space.

\subsection{Translation estimation}

Now assuming we have a good estimate of the rotation, the error in the manual initialization of the translation must be corrected. In that case, there is no need for knowing the whole volume information to estimate the translation: a subvolume, if 


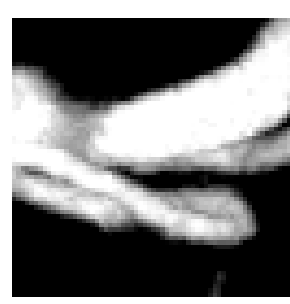

(a) Axial

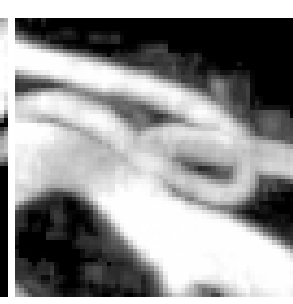

(b) Coronal

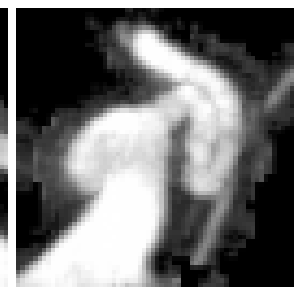

(c) Sagittal

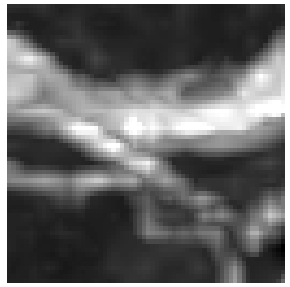

(d) Axial

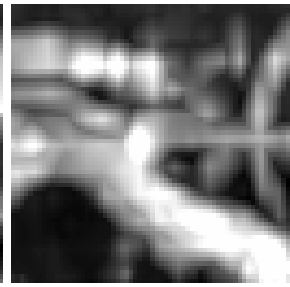

(e) Coronal

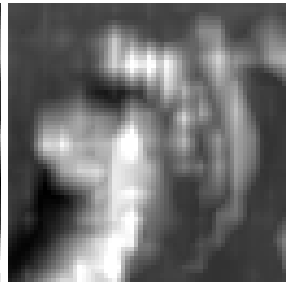

(f) Sagittal

Figure 4: Example results of MIP projections of subcubes in 3DXA (3 on the left) and MR (3 on the right)

correctly chosen, should be enough. Since the homologous pair of point is required to correspond to some artery bifurcation, the local shape of the arteries constraints the translation to a unique value. As a consequence, a sub-cube is extracted surrounding the manually indicated points: the cube dimensions are chosen so that the bifurcation is fully contained in the sub-cube and so that a small error on the rotation can hardly be detected.

Once again, the amount of data is further reduced, while keeping all relevant information regarding arteries, thanks to a MIP projection of the cube along three orthogonal directions: axial, sagittal and coronal. Examples of the images obtained are shown on figure 4. Since the analysis is made locally, the background in MR is rather uniform. This results in very similar images that do not require any pre-treatment.

The similarity criterion we used is the sum of the three cross-correlation scores calculated for the axial, sagittal and coronal images respectively. The translation is recovered when this criterion is maximal.

\subsection{Algorithm scheme}

The Euler angles are used as parameters for the optimization steps of the rotation estimation. The thick shells are defined as lying between spheres centered on the homologous pair of points with radii of $20 \mathrm{~mm}$ and $40 \mathrm{~mm}$. A value of 150 degrees is used for $\varphi_{\max }$. The translation vector is used as parameters for the translation recovery. Sub-cubes of $16 \mathrm{~mm} \times 16 \mathrm{~mm} \times 16 \mathrm{~mm}$ are extracted. The overall algorithm scheme is the following:

- initialization: the user is asked to point the chosen artery bifurcation (internal carotid bifurcation or tip of the basilar artery) in both 3DXA and MR. This provides an initial translation and center points for the rotation. The rotation is estimated through an exhaustive search of the optimal criterion score between low resolution images of the thick shells $(N=64)$ while each angle value vary in $[-30,30]$ degrees each 5 degrees.

- optimization: the translation and rotation are estimated in turn over three loops.

- translation: the similarity criterion is the sum of the three cross-correlation scores between the axial, sagittal and coronal images of the sub-cubes respectively. The cross-correlation scores are established in the Fourier space, which allows for a fast exhaustive optimization of the criterion: translation components are searched for in $[-5,5]$ $\mathrm{mm}$ in a first step, the amplitude being divided by 2 at each loop.

- rotation: the similarity criterion is the normalized centered cross-correlation. The optimization is performed using a hill-climbing scheme also used by Studholme to optimize entropy-based criteria [13], which presents with very interesting convergence time. The initial step for the search is 5 degrees, divided by 2 at each loop. The projected image dimensions increase at each loop from $N=128$ to $N=256$ (last 2 loops).

The average computation time was estimated to be around 30 seconds (not counting the time necessary to load the volumes and manually indicate the initial pair of points), but no systematic measurements were performed since this time appeared to be far below our requirements $(3 \mathrm{mn})$.

Proceedings of SPIE Medical Imaging 2001, K. Janson Ed., 18-22 February 2001, San Diego, vol. 4322 part 1, pp. 623-632. 


\begin{tabular}{|c|c|c|c|}
\hline & Repeatability & Reproducibility & All \\
\hline Average error & 4.48 & 4.94 & 4.85 \\
\hline Standard deviation & 3.08 & 3.75 & 3.62 \\
\hline Maximum error & 11.35 & 17.03 & 17.03 \\
\hline
\end{tabular}

Table 1: Performance of the manual registration as a method of reference (figures are given in millimeters)

\section{Determination of a registration method of reference}

Prior to be able to validate this algorithm, we first need to define a measurement method that enables the comparison of the results of two different registration schemes. Then, a method of reference must be determined. In this regard, two point-based techniques were examined: the first requires the manual definition of pairs of homologous points in both modalities, the second is based on a stereotactic frame.

\subsection{Measurement method}

Since a priori the region of interest where the pathology lies in the images is unknown, we wish to be able to provide an upper limit on the error done over the whole volume of interest. This volume can be limited to the region of support of the 3DXA, which represents an intra-cranial artery and, as such, is always contained inside the region imaged in the MR volume.

As a consequence, the measure of comparison between two registration results is defined as the maximum registration error performed on the 8 corners of the cube enclosing the 3DXA volume (whose side is approximately $15 \mathrm{~cm}$ ). In other words, if the $\left(C_{i}\right)_{i \in\{1 . .8\}}$ are the 8 corners of the cube, the error between two registration results $D_{1}=\left[R_{1} \mid T_{1}\right]$ and $D_{2}=\left[R_{2} \mid T_{2}\right]$ is:

$$
\mathcal{E}\left(D_{1}, D_{2}\right)=\max _{i \in\{1 . .8\}}\left\{\left\|R_{1} C_{i}+T_{1}-R_{2} C_{i}-T_{2}\right\|\right\}
$$

\subsection{Evaluation of the manual registration as a method of reference}

The manual registration allows for retrospective registration and do not require any special setup prior to data acquisition. These properties make such a method very attractive for validation purpose. However, this technique is very prone to error, in particular due to a lack of points and human errors in determining homologous points.

In order to evaluate if the manual method could be trusted in the 3DXA/MR registration case, an experiment was setup:

- 11 pairs of exams (3DXA/MR) were selected which presented with various pathologies (arterio-venous malformations, aneurysms, intra-cranial stenoses);

- three expert users, among which 2 senior neuroradiologists (EK, RA and OL) were asked to perform a manual registration on each case by indicating 5 pairs of homologous points;

- each user was required to perform two manual registrations on each case: a first pass was made over the whole set of 11 patients, then a second pass.

The repeatability was estimated by comparing registrations performed by the same user using the comparison measure defined in the previous paragraph (33 measures). The reproducibility was estimated by comparing registrations performed by different users (396 measures). The average, standard deviations and maximum errors are given in table 1 (in $\mathrm{mm}$ ).

These results clearly show that the manual registration method cannot be used as a method of reference in order to validate an automated algorithm in the 3DXA/MR registration case.

Another interesting conclusion that can be drawn from this experiment is that two matches cannot be distinguished, in the average case, by a human operator, even a trained one, if the maximum registration error is below $5 \mathrm{~mm}$.

Proceedings of SPIE Medical Imaging 2001, K. Janson Ed., 18-22 February 2001, San Diego, vol. 4322 part 1, pp. 623-632. 


\begin{tabular}{|c|c|c|c|c|c|c|c|c|c|c|}
\hline & pat. \#1 & pat. \#2 & pat. \#3 & pat. \#4 & pat. \#5 & pat. \#6 & pat. \#7 & pat. \#8 & pat. \#9 & All \\
\hline Average & 0.046 & 0.061 & 0.011 & 0.084 & 0.085 & 0.077 & 0.018 & 0.017 & 0.034 & 0.048 \\
\hline Std dev & 0.030 & 0.060 & 0.004 & 0.057 & 0.055 & 0.032 & 0.014 & 0.007 & 0.013 & 0.045 \\
\hline Maximum & 0.090 & 0.137 & 0.016 & 0.161 & 0.158 & 0.114 & 0.034 & 0.027 & 0.049 & 0.161 \\
\hline
\end{tabular}

Table 2: Performance of the frame-based registration as a method of reference (figures are given in millimeters)

\begin{tabular}{|l|c|c|c|c|c|c|c|c|c|c|}
\hline & p. \#1 & p. \#2 & p. \#3 & p. \#4 & p. \#5 & p. \#6 & p. \#7 & p. \#8 & p. \#9 & All \\
\hline Error & 1.38 & 1.29 & 1.28 & 1.70 & 1.70 & 1.59 & 1.12 & 1.28 & 1.09 & 1.38 \\
\hline
\end{tabular}

Table 3: Average error on the frame markers at the end of the automatic detection of the frame in MR (figures are given in millimeters)

\subsection{Evaluation of the stereotactic frame as a method of reference}

A database of 9 pairs of 3DXA/MR exams acquired with a stereotactic frame (Leksell frame) was built (8 patients). Automatic methods were used to detect the frame in both 3DXA and MR volumes. Nevertheless, the algorithm used in MR requires the user to point one of the markers on the frame (Leksell frame). As a result, some variability could be expected in that method. In order to assess its extent, one user was asked to perform the frame detection five times on each MR exam. The registration results based on the frame detected were compared for each patient using the measurement defined above (10 measures per patient). The average measure, standard deviation and maximum value are reported on table 2 for each patient and the overall set of measures.

The results are very good: when evaluating a registration method using this frame-based matching technique as a reference, the amount of variation in the results that are due to the imperfections of the reference method do not exceed 0.1 millimeter, which allows for a high significance of an analysis of variance. In conclusion, the frame-based registration method is a good reference method for the 3DXA/MR registration problem.

However, these results do not demonstrate the absolute precision of the frame-based registration technique. Even though the frame-based method has been shown to be repeatable, there might exist some bias or imprecision in the detection of the markers. The distortions in MR are the major concern here. Luckily, the automatic detection algorithm provides us with some hint about the precision of the frame-based registration: the average error found on the frame markers is given as an output. Since the markers are located at the periphery of the head, this measure, though not exactly the same, can be linked with our measurement method. These figures are reported in table 3. These results are not surprising since the values are of the same magnitude as the resolution of the MR acquisitions (between 1 and $1.5 \mathrm{~mm}$ ). However, they also mean that the comparison measure using the frame-based method as a reference has no absolute significance when the error found does not exceed 2 or $3 \mathrm{~mm}$ (the standard deviation of the error on the frame markers would be required to assess this more precisely).

\section{$5 \quad$ Validation of the algorithm}

\subsection{Experimental setup}

In the experiment, we used the same set of data as used to validate the stereotactic frame as a reference method. The initial point was indicated in each 3DXA volume on the predefined artery bifurcation. The homologous point in MR was calculated by applying the rigid-body transform making the match between the 3DXA and MR volumes according to the stereotactic frame. We call this point the ideal point.

A cloud of 50 points was generated for each patient, following a Gaussian random distribution with a standard deviation of $1.5 \mathrm{~mm}$ : at least one point in each cloud was located at more than $5 \mathrm{~mm}$ from the ideal point. These points simulate an expected user error when defining the initial point prior to running the algorithm. The automated registration algorithm was

Proceedings of SPIE Medical Imaging 2001, K. Janson Ed., 18-22 February 2001, San Diego, vol. 4322 part 1, pp. 623-632. 


\begin{tabular}{|c|c|c|c|c|c|c|c|c|c|c|}
\hline & p. \#1 & p. \#2 & p. \#3 & p. \#4 & p. \#5 & p. \#6 & p. \#7 & p. \#8 & p. \#9 & All \\
\hline \# of failures & $27 / 50$ & $6 / 50$ & $1 / 50$ & $33 / 50$ & $2 / 50$ & $0 / 50$ & $0 / 50$ & $16 / 50$ & $0 / 50$ & $85 / 450$ \\
\hline Average error & 7.80 & 3.01 & 2.14 & 3.07 & 3.14 & 3.07 & 2.21 & 5.68 & 2.82 & 3.31 \\
\hline Std dev & 1.39 & 0.19 & 0.15 & 0.28 & 0.43 & 1.82 & 0.28 & 1.54 & 0.26 & 1.74 \\
\hline
\end{tabular}

Table 4: Performances of the automated registration algorithm compared with the stereotactic frame based method. Error measures (average and standard deviation) are given in millimeters and have been calculated by considering only the successful cases.

run for each simulated pair of points on each patient (450 runs) and the result compared to the registration obtained thanks to the stereotactic frame, using our measurement method.

\subsection{Results}

This experiment was designed to assess the performance of the algorithm in terms of precision and robustness.

We evaluated the robustness of the algorithm thanks to two figures. First, following the results on the manual registration evaluation, we defined a success threshold for the algorithm at $10 \mathrm{~mm}$ error: trials leading to an error above $10 \mathrm{~mm}$ are considered to have failed, others are successes. Second, the standard deviation of the error for the successful registrations provides us with a hint about the repeatability of the automated algorithm.

The precision of the algorithm was evaluated through the average error measured on the successful registrations.

Table 4 reports the values obtained for each patient and the overall data set. The algorithm succeeded well for 6 patients out of 9 , for a general success rate of $81.1 \%$. The precision is very good on the successful patients, around $3 \mathrm{~mm}$, and the algorithm robust: the standard deviation is below $0.5 \mathrm{~mm}$ for all successful patients except 1 (patient \#6). If all successful registrations are included, the average error is of $3.31 \mathrm{~mm}$ for a standard deviation of $1.79 \mathrm{~mm}$.

Patient \#1 presents with a failure rate of 54\%. A closer look at these data show a very bad MR image quality, in particular at the carotid bifurcation where the MR signal is almost totally lost. As a result, the translation estimation is perturbed a lot and fails most of the time.

Patients \#4 and \#8 present with a failure rate of $66 \%$ and $32 \%$ respectively. The situation here is different. The images used for the rotation estimation show two very close arteries (anterior cerebral arteries). Since only one of these arteries is imaged in the 3DXA volume, the criterion used for rotation estimation forms a surface with two main local optima (the artery seen in 3DXA is alternatively associated to one or the other anterior cerebral artery shown in MR). The algorithm is unable to differentiate between both optima at low resolution which implies that the initialization of the rotation leads to wrong angle values. In the case of patient \#4, the local optima are further away from each other than for patient \#8 which explains the higher failure rate in the first case, whereas the iterative optimization is able to correct for a wrong initialization in the second case and lower this rate.

Finally, patient \#6 shows a large standard deviation (1.82 $\mathrm{mm}$ with respect to less than $0.5 \mathrm{~mm}$ ). For this patient, 1 failure was reported (error above $10 \mathrm{~mm}$ ) but the data show that 2 measures are above $9 \mathrm{~mm}$, while the remaining 48 measures are below $3.25 \mathrm{~mm}$. This false positive explains the outlier standard deviation.

\section{Discussion}

In the course of this study, we designed an original algorithm, based on the optimization of correlation based similarity measures, which addresses the problems the a priori analysis emphasized:

- initialization: the indication of a homologous pair of points by the user provides an easy way to obtain a pretty good estimate for the translation;

Proceedings of SPIE Medical Imaging 2001, K. Janson Ed., 18-22 February 2001, San Diego, vol. 4322 part 1, pp. 623-632. 
- convergence: the exhaustive search at low resolution on the mappings of the thick shells, enables to get close to the global optimal rotation;

- time: while we did not perform any specific study on the convergence time, the average time for the automated algorithm was below 30 seconds, while the expert users who contributed to the validation of the manual registration as a reference method, stated that a minimum time of 15 minutes was required to perform it on simple cases, with an average time of 30 minutes.

The automated algorithm was evaluated against a frame-based registration method on a set of clinical data ( 9 patients). Our algorithm presented with a good success rate and very good performances in terms of precision and robustness: the maximum registration error is below $4 \mathrm{~mm}$ with a standard deviation of less than $0.5 \mathrm{~mm}$. Giving the intrinsic error estimated on the stereotactic frame arount 1 to $1.5 \mathrm{~mm}$, this result makes our algorithm a valid way to perform 3DXA/MR registration.

As a conclusion, the algorithm we described in this paper is very promising for 3DXA/MR registration. However, our validation procedure pinpointed some remaining issues. The most important one that still needs to be addressed in further improvements concerns the management of local optima in the optimization of the rotation. Two ways are under study. On one hand, the tophat operator perturbs the correlation surface: other high-pass filters should be looked over. On the other hand, the low resolution image is obtained via a bilinear interpolation. Local optima should be better wiped out using Gaussian smoothing filter to lower down the resolution of images.

\section{References}

[1] R. Anxionnat, S. Bracard, J. Macho, E. da Costa, R. Vaillant, L. Launay, Y. Trousset, R. Roméas, and L. Picard, “3D angiography: clinical interest and first applications in interventional radiology," J. of Neuroradiology 25, pp. 251-262, 1998.

[2] R. Anxionnat, S. Bracard, X. Ducrocq, Y. Trousset, L. Launay, E. Kerrien, M. Braun, R. Vaillant, F. Scomazzoni, A. Lebedinsky, and L. Picard, "Clinical value of three-dimensional digital subtraction angiography (3D DSA) in the therapeutic decision and endovascular treatment of intracranial aneurysms," Radiology, 2001. To be published in March.

[3] N. Alperin, D. N. Levin, and C. A. Pelizzari, "Retrospective registration of X-ray angiograms with MR images by using vessels as intrinsic landmarks," J. of Magnetic Resonance Imaging 4, pp. 139-144, March/April 1994.

[4] J. Feldmar, N. Ayache, and F. Betting, "3D-2D projective registration of free form curves and surfaces," Computer Vision and Image Understanding 65(3), pp. 403-424, 1997.

[5] Y. Kita, D. L. Wilson, and J. A. Noble, "Real-time registration of 3D cerebral vessels to X-ray angiograms," in Proc. of MICCAI'98, Cambridge, Massachusetts (USA), W. M. Wells III, A. Colchester, and S. Delp, eds., no. 1496 in LNCS, pp. 1125-1133, Springer Verlag, October 1998.

[6] A. Liu, E. Bullitt, and S. M. Pizer, “3D/2D registration via skeletal near projective invariance in tubular objetcs," in Proc. of MICCAI'98, Cambridge, Massachusetts (USA), W. M. Wells III, A. Colchester, and S. Delp, eds., no. 1496 in LNCS, pp. 952-963, Springer Verlag, October 1998.

[7] E. Kerrien, M.-O. Berger, E. Maurincomme, L. Launay, R. Vaillant, and L. Picard, "Fully automatic 3D/2D subtracted angiography registration," in Proc. of MICCAI'99, Cambridge (UK), C. Taylor and A. Colchester, eds., no. 1679 in LNCS, pp. 664-671, Springer Verlag, September 1999.

[8] K. Krissian, G. Malandain, N. Ayache, R. Vaillant, and Y. Trousset, "Model based detection of tubular structures in 3D images," Tech. Rep. 3736, Institut National de Recherche en Informatique et en Automatique, July 1999. Available on http://www.inria.fr/RRRT/publications-eng.html.

\section{Proceedings of SPIE Medical Imaging 2001, K. Janson Ed., 18-22 February 2001, San Diego, vol. 4322 part 1 , pp. 623-632.}


[9] D. L. Wilson and J. A. Noble, "An adaptive segmentation algorithm for time-of-flight MRA data," IEEE Trans. on Medical Imaging 18(10), pp. 938-945, 1999.

[10] A. F. Frangi, W. J. Niessen, R. M. Hoogeveen, T. van Walsum, and M. A. Viergever, "Model-based quantitation of 3-D magnetic resonance angiographic images," IEEE Trans. on Medical Imaging 18(10), pp. 946-956, 1999.

[11] A. C. Chung, J. A. Noble, and P. Summer, "Fusing speed and phase information for vascular segmentation in phase contrast MR angiograms," in Proc. of MICCAI'00, Pittsburgh, Pennsylvania (USA), A. M. D. S. L. Delp and B. Jaramaz, eds., no. 1935 in LNCS, pp. 166-175, Springer Verlag, October 2000.

[12] A. Collignon, F. Maes, D. Delaere, D. Vandermeulen, P. Suetens, and G. Marchal, "Automated multi-modality image registration based on information theory," in Proc. of IPMI'95, Ile de Berder (France), Y. B. et al, ed., pp. 263-274, Kluwer Academic Publishers, June 1995.

[13] C. Studholme, D. L. G. Hill, and D. J. Hawkes, "Automated 3D registration of MR and CT images of the head," Medical Image Analysis 1(2), pp. 163-175, 1996.

[14] J. West, J. M. Fitzpatrick, M. Y. Wang, B. M. Dawant, C. R. Maurer Jr, R. M. Kessler, R. J. Maciunas, C. Barillot, D. Lemoine, A. Collignon, F. Maes, P. Suetens, D. Vandermeulen, P. A. van den Elsen, S. Napel, T. S. Sumanaweera, B. Harkness, P. F. Hemler, D. L. G. Hill, D. J. Hawkes, C. Studholme, J. B. A. Maintz, M. A. Viergever, G. Malandain, X. Pennec, M. E. Noz, G. Q. Maguire, M. Pollack, C. A. Pelizzari, R. A. Robb, D. Hanson, and R. P. Woods, "Comparison and evaluation of retrospective intermodality brain image registration techniques," J. of Computer Assisted Tomography 21, pp. 554-566, July/August 1997.

[15] A. Roche, G. Malandain, N. Ayache, and S. Prima, "Towards a better comprehension of similarity measures used in medical image registration," in Proc. of MICCAI'99, Cambridge (UK), C. Taylor and A. Colchester, eds., no. 1679 in LNCS, pp. 555-566, Springer Verlag, September 1999. 